\title{
Editorial
}

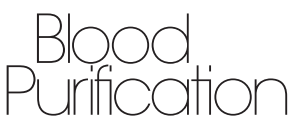

\section{SIKD Is On the Road}

\author{
Xiaoqiang Ding \\ Department of Nephrology, Zhongshan Hospital, Shanghai Medical College, Fudan University, Shanghai Institute \\ for Kidney and Dialysis (SIKD), Shanghai Key Laboratory of Kidney and Blood Purification, Shanghai, China
}

The Department of Nephrology, Zhongshan Hospital, Fudan University, Shahnghai, China (fig. 1), authorized with key discipline by National Ministry of Education, is an important base for treatments, research works, teaching and training in kidney disease. It is also called the Shanghai Institute for Kidney and Dialysis (SIKD). Our department consists of 4 parts: outpatients' service, inpatients' ward, blood purification center and kidney disease laboratory. Being a pioneer in conducting hemodialysis technology in China, the department has tremendous clinical experience and profound research force in the fields of blood purification, glomerular disease, chronic kidney disease, acute kidney injury and renal replacement in critical disease. Today we have not only acquired a leading position in China but also earned international reputations.

\section{History}

Our passed Prof. Lvtan Liao was the founder of hemodialysis in China and we are one of the first institutions which start dialysis since 1956. The historic events in our department are as follows:

(1) In 1956, we conducted the first hemodialysis for acute renal failure in China.

(2) In 1973, our department participated in the invention of the first flat type hemodialysis machine in China and conducted the first practice of hemodialysis in China (fig. 2).

(3) We introduced a series of new technologies into the clinical practice in the following years: first peritoneal dialysis practice (1966), first hemodialysis practice for treating uremia (1973), hemoperfusion (1978), continuous renal replacement in treating critical acute renal failure (1984), optimized continuous renal replacement therapy (CRRT) practice (non-nocturnal CRRT in 1994, highflux hemofiltration in 1999, prolonged daily hemofiltration in 2000), citric acid hemodialysis (1995), dual plasmapheresis (1999), molecular absorbent recycling system (MARS) artificial liver (2001), protein A immunoadsorption (2003), combination with ECOM and CRRT (2008). Currently, these technologies have been popularized in the nation and are playing critical roles in the clinical practice.

(4) In 1983, we first reported the non-oliguria acute renal failure and acute progressive glomerulonephritis in China.

(5) In 2002, we were authorized to be the key discipline by the National Ministry of Education.

(6) In 2014, we were authorized to be Shanghai Institute for Kidney and Dialysis (SIKD), and Shanghai Key Laboratory of Kidney and Blood Purification.

(7) In 2015, we become the Quality Control Center of Dialysis in Shanghai.

\section{KARGER}

E-Mail karger@karger.com www.karger.com/bpu
(C) 2016 S. Karger AG, Basel

0253-5068/16/0421-000I\$39.50/0
Xiaoqiang Ding

Department of Nephrology, Zhongshan Hospital

Shanghai Medical College, Fudan University

Shanghai Institute for Kidney and Dialysis (SIKD), Shanghai (China)

E-Mail ding.xiaoqiang@zs-hospital.sh.cn 
Fig. 1. The building of the Shanghai Zhongshan Hospital.

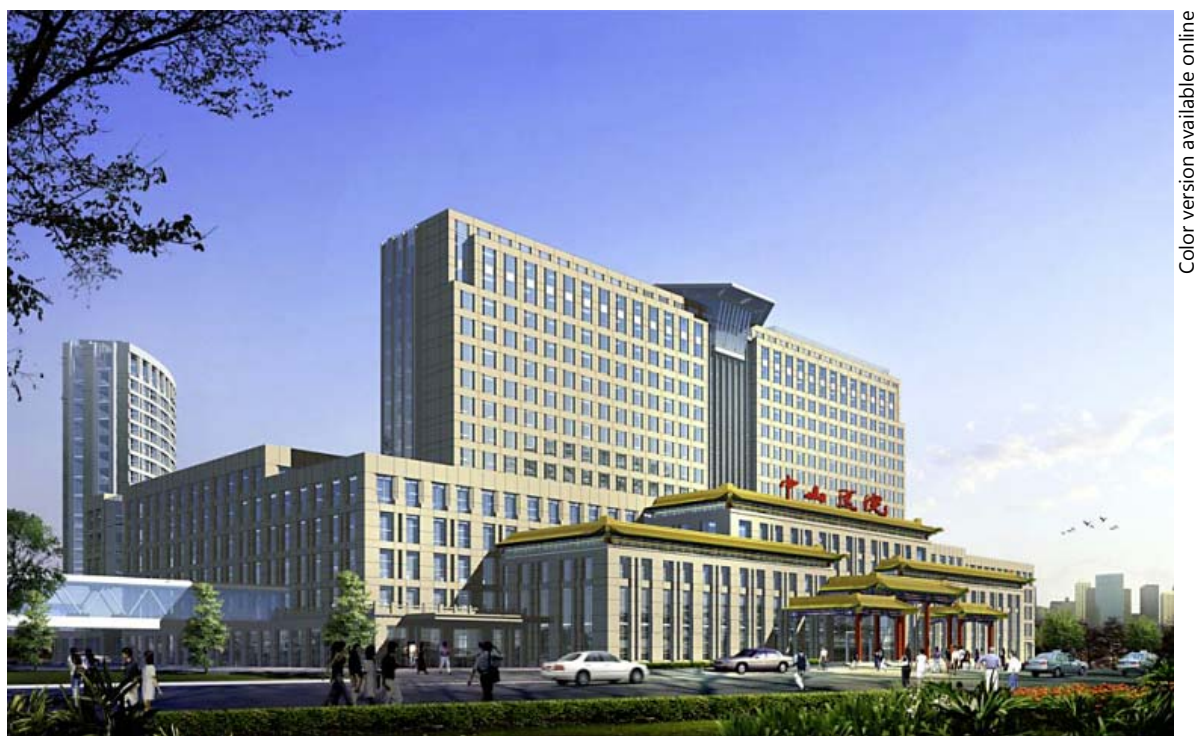

Fig. 2. The first flat-type hemodialysis machine in China from Department of $\mathrm{Ne}$ phrology of Shanghai Zhongshan Hospital.

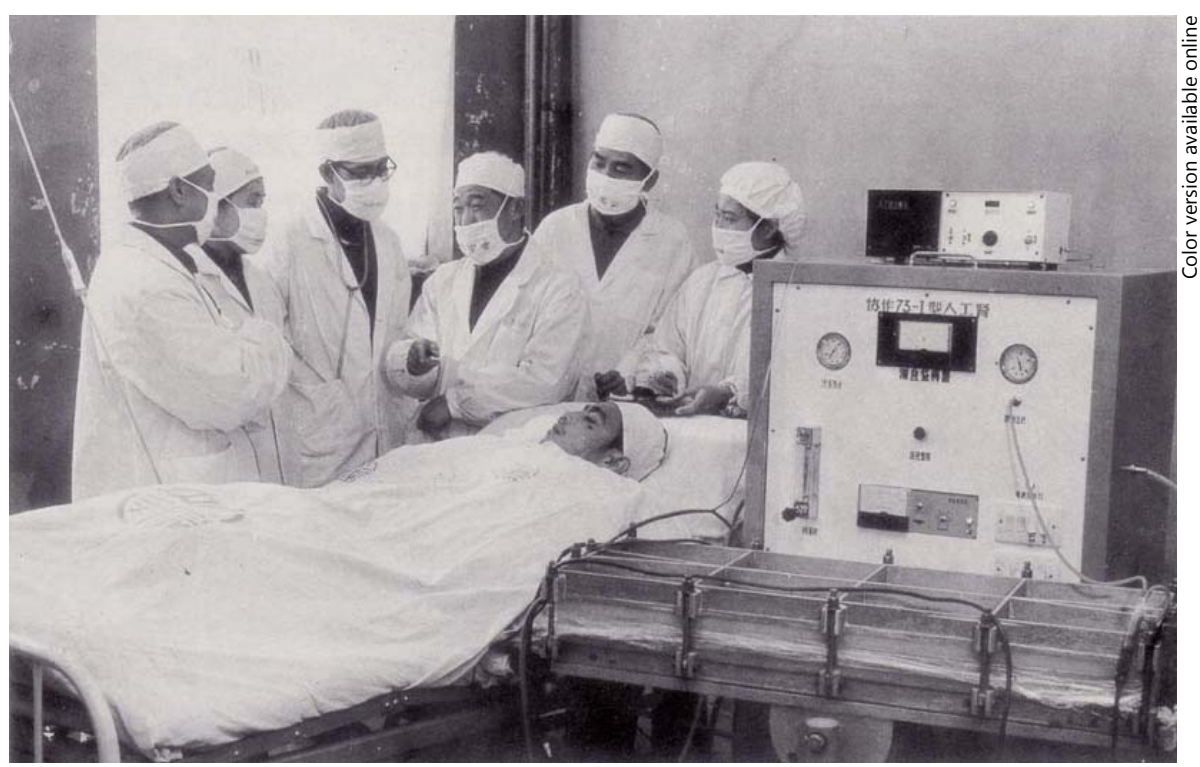

\section{Faculty and Clinical Practice}

At present we have 111 faculties in service, 37 doctors including 7 professors, 7 associate professors, 21 attending doctors and 12 technicians, and more than 60 nurses. Many of them are members of influential academic associations.

Our department serves as a major renal disease center in China. We have received a large number of patients on whom diagnosis and application of therapy have been difficult. Our outpatient division together with emergency di- vision and consultation services handle more than 200,000 patients per year, which include 50,000 patients seeking expert services. The inpatient division has 80 beds, which services 3,500 patients from around the country every year (fig. 3). We offer various special outpatient and inpatient services, including treatment for diabetic nephropathy, hypertension nephropathy, urinary tract infection, peritoneal dialysis, uremia, hyperuricemia, gouty nephropathy, lupus nephritis, edema and nutrition in kidney disease and renal biopsy follow-up. We ensure the patients have sufficient and easy access to doctors and services. 
Fig. 3. The routine ward inspection of SIKD.

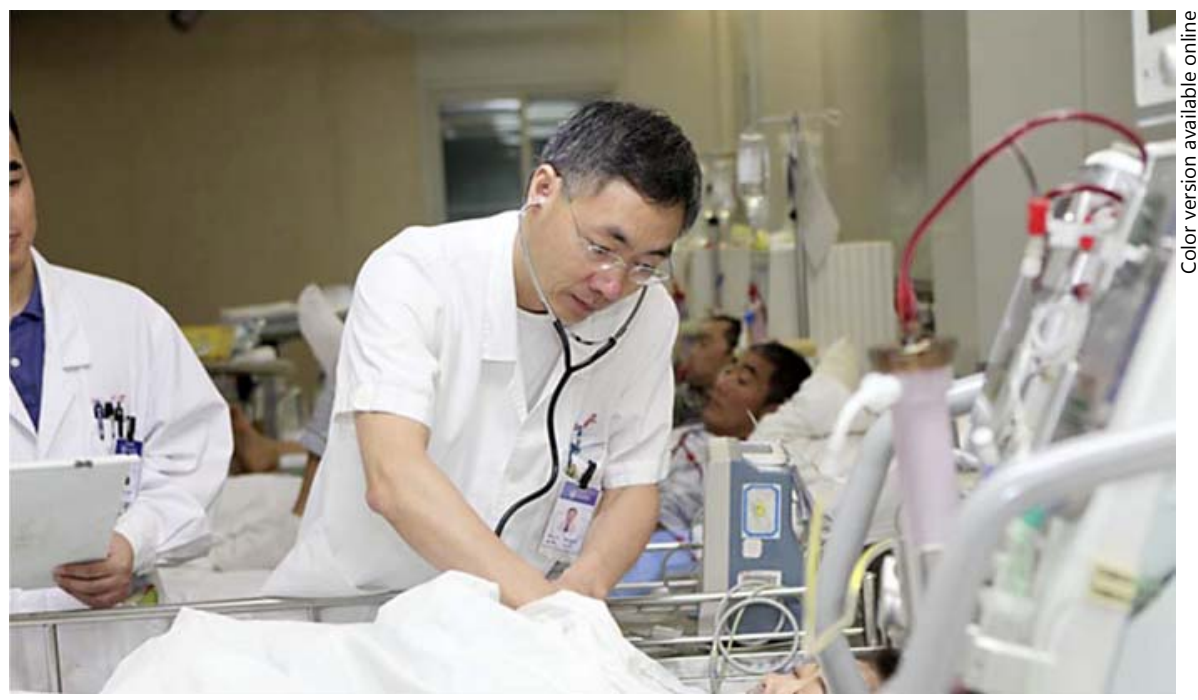

Fig. 4. The modern building of the hemodialysis center.

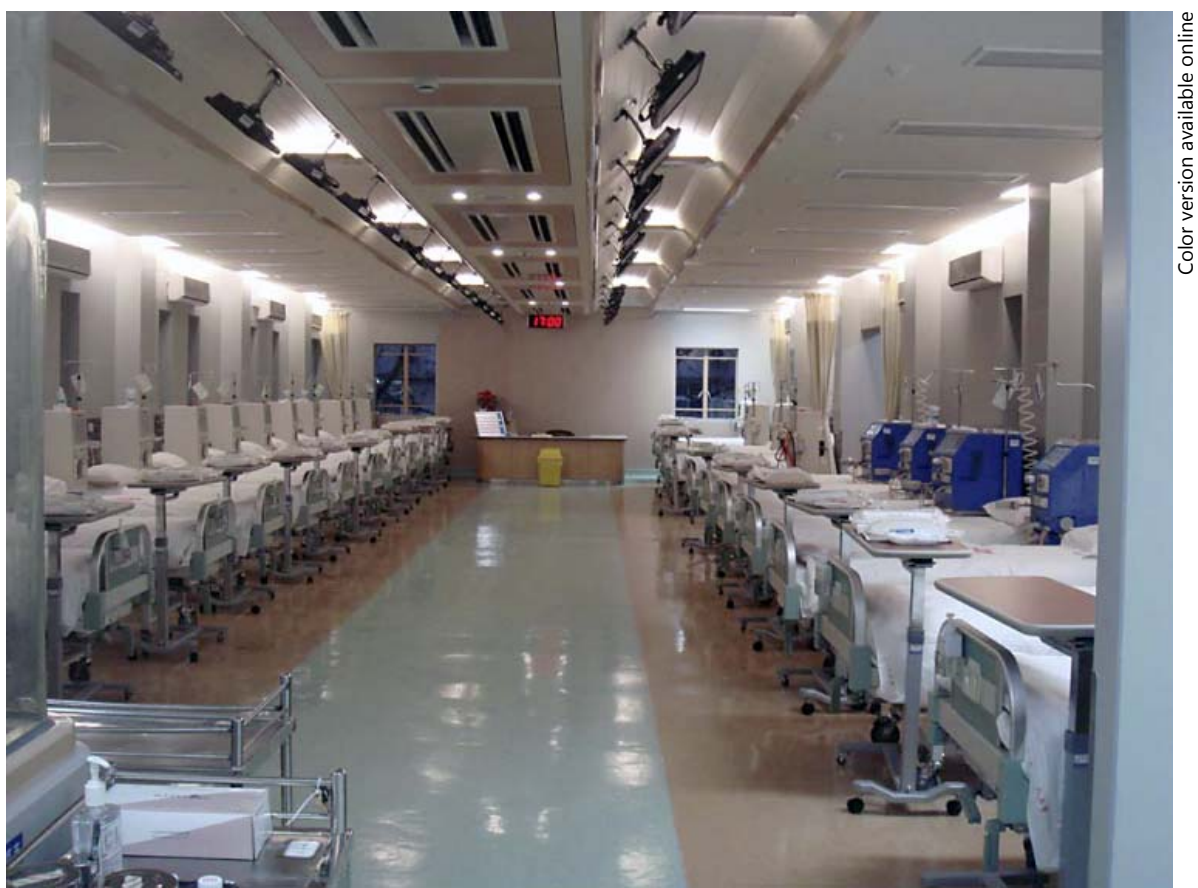

The blood purification center possesses a $1,800 \mathrm{~m}^{2}$ therapy room, which is equipped with nearly 120 most advanced hemodialysis and CRRT machines (fig. 4). The center serves 520 patients on maintenance hemodialysis, which makes it one of the largest and most comprehensive domestic blood purification centers. At the same time, we treat a considerable number of patients with severe or refractory uremia for temporary dialysis from all around the country every year. The long survival rate of our patients on maintenance hemodialysis has reached the advanced survival rates in the world.

We also have nearly 20 CRRT machines for critical patients in ICU, which serves nearly 200 sessions every month. As for peritoneal dialysis, we have more than 200 patients who are on peritoneal dialysis with various patterns.

The blood purification center and kidney disease laboratory (fig. 5) are the two important divisions where 
Fig. 5. The laboratories of SIKD.

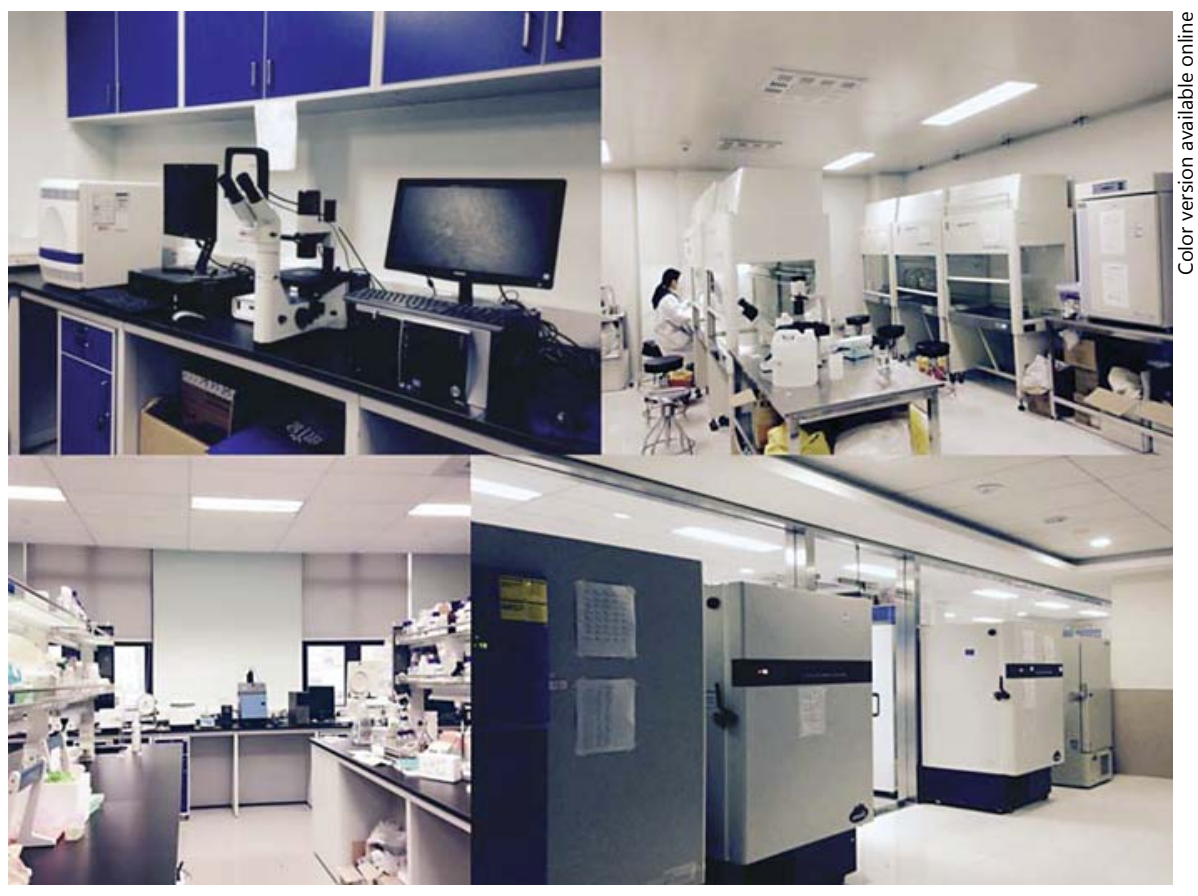

Fig. 6. The Shanghai Forum on Acute Kidney Injury (AKI 2014) in Shanghai.

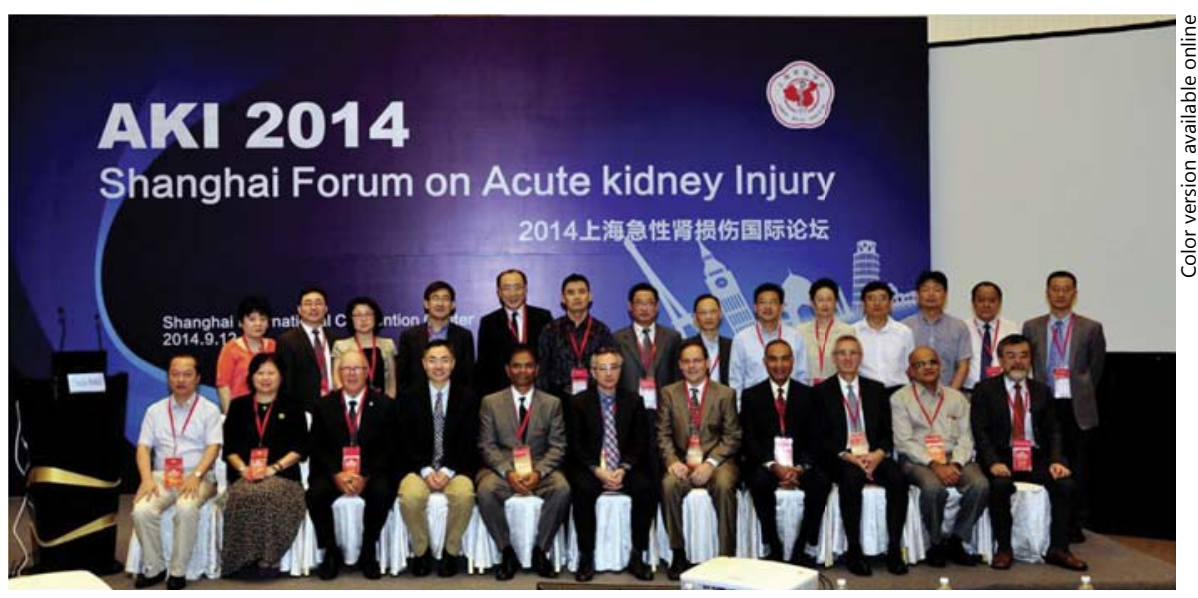

intense scientific researches are conducted. Our research areas include renal pathophysiology, prevention of acute kidney injury, chronic kidney disease, and new therapy in blood purification and translational medicine for diagnosis and therapy. We were in charge of 14 key projects and 16 clinical RCTs, and those results were published in the highest-level journals in the field of nephrology. Last year, we were authorized to be the Key Laboratory of Kidney and Blood Purification, and became the Quality Control Center of Dialysis in Shanghai.

\section{Medical Education}

Our department has produced the first batch of doctors in nephrology in China. Till now, 53 students have graduated from our department with doctor degrees, 89 with master degrees, and 3 with post-doctoral degrees. Every year, a large number of doctors from 30 provinces in China come here to pursue advanced studies, and more than 100 of them receive the degrees. Our doctors also take part in compiling textbooks and the following were compiled by our doctors: the Textbook 
Fig. 7. Distinguished Professor Claudio Ronco visits SIKD.

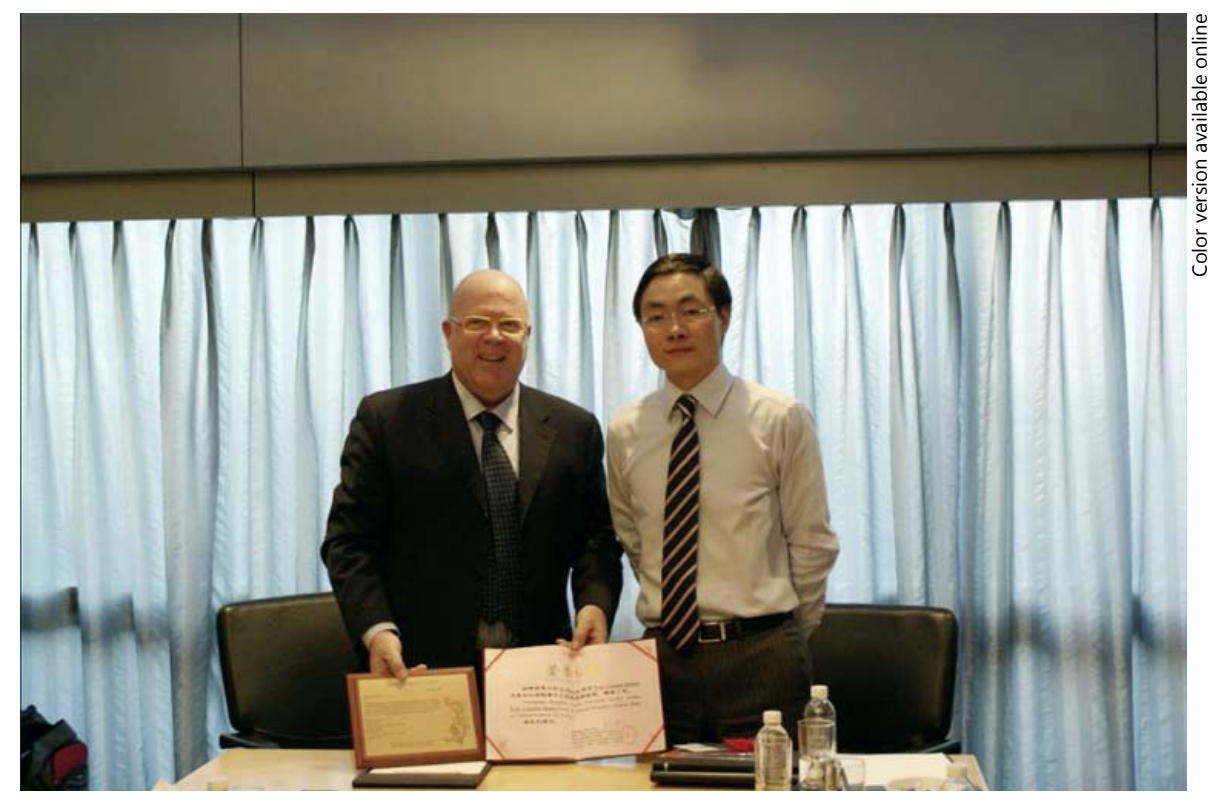

Fig. 8. A group of young members of SIKD.

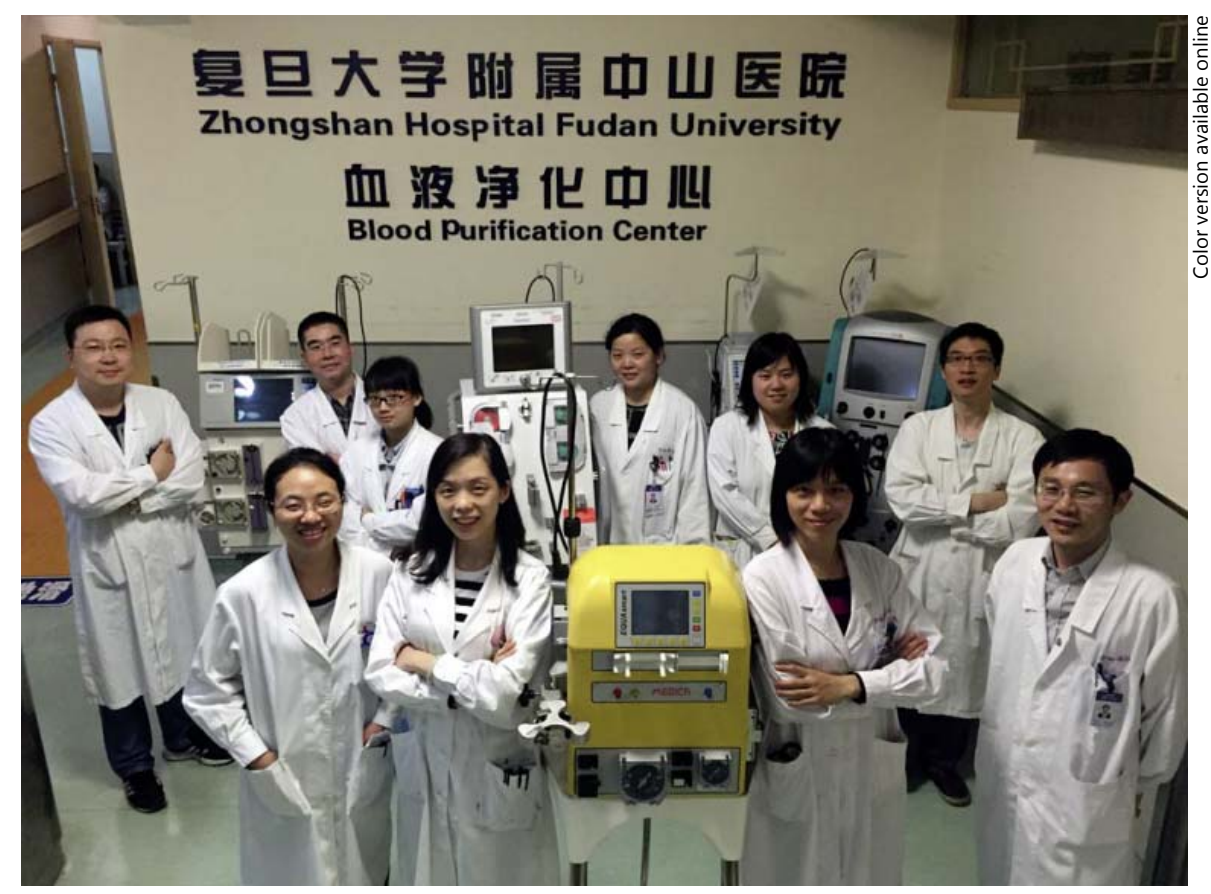

of Internal Medicine, Encyclopedia of Chinese Medicine (chapter on hemodialysis), and Pharmacopeia of the People's Republic of China (chapter on diuretics and drug of water, electrolytes and acid-base balance 1st and 2 nd edition).

\section{Academics Activities}

Since 1985, our department has held more than 50 academic conferences and Continuing Medical Education courses on hemodialysis, diagnosis and treatment of kidney disease. It has been estimated that over 30,000 par- 
ticipants have attended these conferences and been part of the courses. Since 2013, we started collaborating with the International Congress of Society for Hemodialysis to hold international conferences for hemodialysis in Shanghai, which have strongly enhanced the quality of hemodialysis in China (fig. 6, 7).

We took part in compiling the Standard of Procedure of hemodialysis, drafting the Standard of Procedure of blood purification, clinical routine for AKI, and expert consensus on glucocorticoid in kidney disease $(2008,2010)$ by Chinese Society of Nephrology. We are proud to be the chief editor of the Clinical Practice
Standards - Nephrology Session (1996) and standards of diagnosis and treatment of kidney disease authorized by Shanghai Health Bureau and Institution of Medicine.

We are honored that the experts in our department have made many contributions to policy making by providing professional consultations in many significant events. I believe our efforts will help promote the popularization of kidney disease prevention, and early diagnoses and interference around the country. All the members of SIKD will continue working for this purpose, and SIKD is always moving forward on this road (fig. 8). 